\title{
Car-Sharing between Two Locations: Online Scheduling with Flexible Advance Bookings
}

\author{
Kelin Luo ${ }^{1,2}$, Thomas Erlebach ${ }^{2}$, and Yinfeng $\mathrm{Xu}^{1}$ \\ 1 School of Management, Xi'an Jiaotong University, China \\ 2 Department of Informatics, University of Leicester, United Kingdom
}

\begin{abstract}
We study an on-line scheduling problem that is motivated by applications such as car-sharing. Users submit ride requests, and the scheduler aims to accept requests of maximum total profit using a single server (car). Each ride request specifies the pick-up time and the pick-up location (among two locations, with the other location being the destination). The scheduler has to decide whether or not to accept a request immediately at the time when the request is submitted (booking time). We consider two variants of the problem with respect to constraints on the booking time: In the fixed booking time variant, a request must be submitted a fixed amount of time before the pick-up time. In the variable booking time variant, a request can be submitted at any time during a certain time interval that precedes the pick-up time. We present lower bounds on the competitive ratio for both variants and propose a greedy algorithm that achieves the best possible competitive ratio.
\end{abstract}

\section{Introduction}

In a car-sharing system, a company offers cars to customers for a period of time. Customers can pick up a car in one location, drive it to another location, and return it there. Car booking requests arrive on-line, and the goal is to maximize the profit obtained from satisfied requests. We consider a setting where all driving routes go between two fixed locations, but can be in either direction. For example, the two locations could be a residential area and a nearby shopping mall or central business district. Other applications that provide motivation for the problems we study include taxi dispatching and boat rental for river crossings.

In real life, customer requests for car bookings usually arrive over time, and the decision about each request must be made immediately, without knowledge of future requests. This gives rise to an on-line problem that bears some resemblance to interval scheduling, but in which additionally the pick-up and drop-off locations play an important role: The server that serves a request must be at the pick-up location at the start time of the request and will be located at the drop-off location at the end time of the request. A server can serve two consecutive requests only if the drop-off location of the first request is the same as the pick-up location of the second request, or if there is enough time to travel between the two locations otherwise. (We allow 'empty movements' that allow a server to be moved from one location to another while not serving a request. 
Such empty movements could be implemented by having company staff drive a car from one location to another, or in the future by self-driving cars.)

An important aspect of the problem is the relation between the booking time, i.e., the time when the request is submitted, and the start time of the request, i.e., the time when the customer picks up the car at the pick-up location. Constraints on the booking time (also called the reservation window in the context of advance reservation systems) can affect the performance of a system [7]. There are generally two types of bookings, current and advance. Current bookings are requests that are released and must be served immediately. Advance bookings are requests that are released before the start time. In this paper we consider advance bookings. More specifically, we study two variants of advance bookings: In the fixed booking time variant, the amount of time between the booking time of a request and its start time is a fixed value, independent of the request. In the variable booking time variant, the booking time of a request must lie in a certain time interval (called the booking horizon) before the start time of the request.

We assume that every request is associated with a profit that is obtained if the request is accepted. When a server moves from one location to another while not serving a request, a certain cost is incurred. The goal is to maximize the total profit, which is the sum of the profits of the accepted requests minus the costs incurred for moving servers while not serving a request. In this paper, we focus on the special case of a single server.

\subsection{Related Work}

The car sharing problem considered in this paper belongs to the class of dynamic pickup and delivery problems surveyed by Berbeglia et al. [2]. The problem that is closest to our setting is the on-line dial-a-ride problem (OLDARP) that has been studied widely. In OLDARP, transportation requests between locations in a metric space arrive over time, but typically it is assumed that requests want to be served 'as soon as possible' rather than at a specific time as in our problem. Known results for OLDARP include on-line algorithms for minimizing the makespan [1,3] or the maximum flow time [8]. Work on versions of OLDARP where not all requests can be served includes competitive algorithms for requests with deadlines where each request must be served before its deadline or rejected [9], and for settings with a given time limit where the goal is to maximize the revenue from requests served before the time limit [6]. In contrast to existing work on OLDARP, in this paper we consider requests that need to be served at a specific time that is specified by the request when it is released.

Off-line versions of car-sharing problems are studied by Böhmová et al. [4]. They show that if all customer requests for car bookings are known in advance, the problem of maximizing the number of accepted requests can be solved in polynomial time using a minimum-cost network flow algorithm. Furthermore, they consider the problem variant with two locations where each customer requests two rides (in opposite directions) and the scheduler must accept either both or neither of the two. They prove that this variant is NP-hard and APXhard. In contrast to their work, we consider the on-line version of the problem. 


\subsection{Problem Description and Preliminaries}

We consider a setting with only two locations (denoted by 0 and 1 ) and a single server. The travel time from 0 to 1 is the same as the travel time from 1 to 0 and is denoted by $t$. Let $R$ denote a sequence of requests that are released over time. The $i$-th request is denoted by $r_{i}=\left(\tilde{t}_{r_{i}}, t_{r_{i}}, p_{r_{i}}\right)$ and is specified by the booking time or release time $\tilde{t}_{r_{i}}$, the start time $t_{r_{i}}$, and the pick-up location $p_{r_{i}} \in\{0,1\}$. Requests with the same release time arrive one by one in arbitrary order, and each request must be processed by the algorithm before the next request arrives. If $r_{i}$ is accepted, the server must pick up the customer at $p_{r_{i}}$ at time $t_{r_{i}}$ and drop off the customer at location $\dot{p}_{r_{i}}=1-p_{r_{i}}$ at time $\dot{t}_{r_{i}}=t_{r_{i}}+t$, the end time of the request. We say that the request $r_{i}$ starts at time $t_{r_{i}}$. For an interval $[b, d)$, we say that $r_{i}$ starts in the interval if $t_{r_{i}} \in[b, d)$.

The server can only serve one request at a time. Serving a request yields profit $r>0$. The server is initially located at location 0 . If the pick-up location $p_{r_{i}}$ of a request $r_{i}$ is different from the current location of the server and if at least $t$ time units remain before the start time of $r_{i}$, the server can move from its current location to $p_{r_{i}}$. We refer to such moves (which do not serve a request) as empty moves. An empty move takes time $t$ and incurs a cost of $c, 0 \leq c \leq r$, and we say that $r_{i}$ is accepted with cost in this case. If the server is already located at $p_{r_{i}}$, we say that $r_{i}$ is accepted without cost. We forbid 'unprompted' moves, i.e., the algorithm is allowed to make an empty move to the other location only if it does so in order to serve a request that was accepted before the current time and whose pick-up location is the other location. If two requests are such that they cannot both be served by one server, we say that the requests are in conflict.

We denote the requests accepted by an algorithm by $R^{\prime}$, and the $i$-th request in $R^{\prime}$, in order of request start times, is denoted by $r_{i}^{\prime}$. We say that request $r_{i}^{\prime}$ is accepted without cost if $i=1$ and $p_{r_{i}^{\prime}}=0$ or if $i>1$ and $p_{r_{i}^{\prime}}=\dot{p}_{r_{i-1}^{\prime}}$. Otherwise, $r_{i}^{\prime}$ is accepted with cost. We denote the profit of serving the requests in $R^{\prime}$ by $P_{R^{\prime}}$. If $R_{c}^{\prime}$ denotes the subset of $R^{\prime}$ consisting of the requests that are accepted with cost, we have $P_{R^{\prime}}=r \cdot\left|R^{\prime}\right|-c \cdot\left|R_{c}^{\prime}\right|$.

The goal is to accept a set of requests $R^{\prime}$ that maximizes the profit $P_{R^{\prime}}$. The problem for one server and two locations for the fixed booking time variant in which $t_{r_{i}}-\tilde{t}_{r_{i}}=a$ for all requests $r_{i}$, where $a \geq 0$ is a constant, is called the $1 S 2 L-F$ problem. For the variable booking time variant, the booking time $\tilde{t}_{r_{i}}$ of any request $r_{i}$ must satisfy $t_{r_{i}}-b_{u} \leq \tilde{t}_{r_{i}} \leq t_{r_{i}}-b_{l}$, where $b_{l}$ and $b_{u}$ are constants, with $b_{l} \leq b_{u}$, that specify the minimum and maximum length, respectively, of the time interval between booking time and start time. The problem for one server and two locations for the variable booking time variant is called the $1 S 2 L-V$ problem. If $b_{l}=b_{u}$, the $1 \mathrm{~S} 2 \mathrm{~L}-\mathrm{V}$ problem turns into the 1S2L-F problem.

The performance of an algorithm for $1 \mathrm{~S} 2 \mathrm{~L}-\mathrm{F}$ or $1 \mathrm{~S} 2 \mathrm{~L}-\mathrm{V}$ is measured using competitive analysis (see [5]). For any request sequence $R$, let $P_{R^{A}}$ denote the objective value produced by an on-line algorithm $A$, and $P_{R^{*}}$ that obtained by an optimal scheduler $O P T$ that has full information about the request sequence in advance. Like for the algorithm, we also require that $O P T$ does not make 
unprompted moves, i.e., OPT is allowed to make an empty move starting at time $t_{0}$ only if there is an accepted request $r_{i}$ with $\tilde{t}_{r_{i}} \leq t_{0}$ and $t_{r_{i}} \geq t_{0}+t$ whose pick-up location is the other location. Without this restriction on OPT, it would not be possible to achieve finite competitive ratio in cases where a request can be booked less than $t$ units of time before its starting time.

The competitive ratio of $A$ is defined as $\rho_{A}=\sup _{R} \frac{P_{R^{*}}}{P_{R^{A}}}$. We say that $A$ is $\rho$-competitive if $P_{R^{*}} \leq \rho \cdot P_{R_{A}}$ for all request sequences $R$. Let $O N$ be the set of all on-line algorithms for a problem. A value $\beta$ is a lower bound on the best possible competitive ratio if $\rho_{A} \geq \beta$ for all $A$ in $O N$. We say that an algorithm $A$ is optimal if there is a lower bound $\beta$ with $\rho_{A}=\beta$.

\subsection{Paper Outline}

In Section 2, we study the $1 \mathrm{~S} 2 \mathrm{~L}-\mathrm{F}$ problem. We give lower bounds and propose a greedy algorithm that achieves the best possible competitive ratio. In Section 3, we study the $1 \mathrm{~S} 2 \mathrm{~L}-\mathrm{V}$ problem. Although variable booking times provide much greater flexibility to customers, we show that our greedy algorithm is still optimal. Some proofs are omitted due to space restrictions. An overview of our results is shown in Table 1.

Table 1. Lower and upper bounds for the car sharing problem

\begin{tabular}{llllll}
\hline \multicolumn{5}{c}{$0 \leq c<r$} & \multicolumn{3}{c}{$c=r$} \\
\hline Problem & Booking constraint & LB & UB & LB & UB \\
\hline 1S2L-F & $0 \leq a<t$ & 1 & 1 & 1 & 1 \\
1S2L-F & $t \leq a$ & $\frac{2 r}{r-c}$ & $\frac{2 r}{r-c}$ & 1 & 1 \\
1S2L-V & $0<b_{u}<t$ & 3 & 3 & 3 & 3 \\
1S2L-V & $b_{u}=t$ & $\max \left\{\frac{2 r}{r-c}, 3\right\}$ & $\max \left\{\frac{2 r}{r-c}, 3\right\}$ & 3 & 3 \\
1S2L-V & $t<b_{u}$ & $\frac{3 r-c}{r-c}$ & $\frac{3 r-c}{r-c}$ & $1+2\left\lceil\frac{b_{u}-b_{l}}{2 t}\right\rceil$ & $1+2\left\lceil\frac{b_{u}-b_{l}}{2 t}\right\rceil$ \\
\hline
\end{tabular}

\section{Car Sharing with Fixed Booking Times}

In this section, we study the $1 \mathrm{~S} 2 \mathrm{~L}-\mathrm{F}$ problem. First, we present a lower bound. We use $A L G$ to denote any on-line algorithm and $O P T$ to denote an optimal scheduler. We refer to the server of $A L G$ and $O P T$ as $s^{\prime}$ and $s^{*}$, respectively. The set of requests accepted by $A L G$ is referred to as $R^{\prime}$, and the set of requests accepted by OPT as $R^{*}$.

Theorem 1. For $0 \leq c<r$ and $a \geq t$, no deterministic on-line algorithm for $1 S 2 L-F$ can achieve competitive ratio smaller than $\frac{2 r}{r-c}$.

Proof. Initially, the adversary releases a request $r_{1}=(0, a, 1)$. We distinguish two cases. 
Case 1: $A L G$ accepts $r_{1}$ (with cost). The adversary releases requests $r_{2}=$ $(\varepsilon, a+\varepsilon, 0)$ and $r_{3}=(\varepsilon+t, a+\varepsilon+t, 1)$, where $0<\varepsilon<t . O P T$ accepts $r_{2}$ and $r_{3}$ without cost, but $A L G$ cannot accept either of these requests as they are in conflict with $r_{1}$. We have $P_{R^{*}}=2 r$ and $P_{R^{\prime}}=r-c$, and hence $\frac{P_{R^{*}}}{P_{R^{\prime}}}=\frac{2 r}{r-c}$.

Case 2: $A L G$ does not accept request $r_{1}$. In this case, OPT accepts $r_{1}$ and we have $P_{R^{*}}=r-c$ and $P_{R^{\prime}}=0$, and hence $\frac{P_{R^{*}}}{P_{R^{\prime}}}=\infty$.

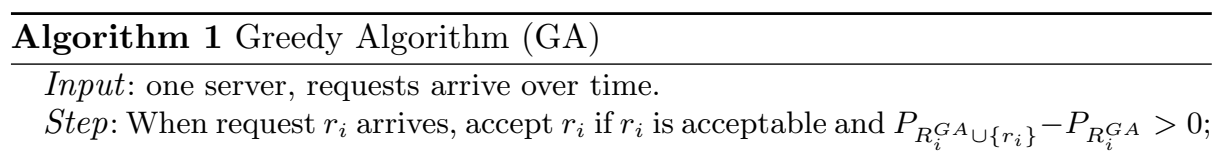

Note 1. $R_{i}^{G A}$ is the set of requests accepted by GA before $r_{i}$ is released.

Note 2. $r_{i}$ is acceptable if and only if $\forall r_{j}^{\prime} \in R_{i}^{G A},\left|t_{r_{i}}-t_{r_{j}^{\prime}}\right| \geq 2 t$ if $p_{r_{i}}=p_{r_{j}^{\prime}}$, and $\left|t_{r_{i}}-t_{r_{j}^{\prime}}\right| \geq t$ if $p_{r_{i}} \neq p_{r_{j}^{\prime}}$, and $t_{r_{i}}-\tilde{t}_{r_{i}} \geq t$ if $s^{\prime}$ is at location $p_{s^{\prime}} \in\{0,1\}$ at time $\tilde{t}_{r_{i}}$ and $p_{r_{i}}=1-p_{s^{\prime}}$.

We propose a Greedy Algorithm (GA) for the 1S2L-F problem, shown in Algorithm 1. For an arbitrary request sequence $R=\left\{r_{1}, r_{2}, r_{3}, \ldots, r_{n}\right\}$, note that we have $t_{r_{i}} \leq t_{r_{i+1}}$ for $1 \leq i<n$ because $t_{r_{i}}-\tilde{t}_{r_{i}}=a$ is fixed. Denote the requests accepted by OPT by $R^{*}=\left\{r_{1}^{*}, r_{2}^{*}, \ldots, r_{k^{*}}^{*}\right\}$ and the requests accepted by GA by $R^{\prime}=\left\{r_{1}^{\prime}, r_{2}^{\prime}, \ldots, r_{k}^{\prime}\right\}$ indexed in order of non-decreasing start times.

Theorem 2. Algorithm GA is 1-competitive for $1 S 2 L-F$ if $c=r$, or if $0 \leq c<r$ and $0 \leq a<t$.

Proof. If $0 \leq c<r$ and $0 \leq a<t$, GA and $O P T$ only accept requests without cost because the release time of a request is too late for the server to be able to serve it with cost (recall that we forbid unprompted moves by $O P T$ ). Observe that this means that both GA and $O P T$ accept requests with alternating pick-up location, starting with a request with pick-up location 0 .

We claim that $R^{*}$ can be transformed into $R^{\prime}$ without reducing its profit, thus showing that $P_{R^{*}}=P_{R^{\prime}}$. As GA accepts the first request $r_{j}$ with $p_{r_{j}}=0$, it is clear that $t_{r_{1}^{\prime}} \leq t_{r_{1}^{*}}$. If $r_{1}^{\prime} \neq r_{1}^{*}$, we can replace $r_{1}^{*}$ by $r_{1}^{\prime}$ in $R^{*}$, and $R^{*}$ is still a valid solution with the same profit. Now assume, that $R^{\prime}$ and $R^{*}$ are identical with respect to the first $i$ requests, and that $s^{\prime}$ and $s^{*}$ are at location $p \in\{0,1\}$ at time $\dot{t}_{r_{i}^{\prime}}$. If there is a request $r_{i+1}^{*}$, there must also be a request $r_{i+1}^{\prime}$ with $t_{r_{i+1}^{\prime}} \leq t_{r_{i+1}^{*}}$, as GA could accept $r_{i+1}^{*}$. We can replace $r_{i+1}^{*}$ by $r_{i+1}^{\prime}$ in $R^{*}$. The claim thus follows by induction.

If $c=r$, accepting a request with cost yields profit $r-c=0$. Without loss of generality, we can therefore assume that both GA and $O P T$ only accept requests without cost. The arguments of the previous paragraph can then be applied to this case as well. 
Theorem 3. Algorithm GA is $\frac{2 r}{r-c}$-competitive for $1 S 2 L-F$ if $0 \leq c<r$ and $a \geq t$.

Proof. We partition the time horizon $[0, \infty)$ into intervals (periods) that can be analyzed independently. Period $i$, for $1<i<k$, is the interval $\left[t_{r_{i}^{\prime}}, t_{r_{r^{\prime}+1}}\right)$. Period 1 is $\left[0, t_{r_{2}^{\prime}}\right)$, and period $k$ is $\left[t_{r_{k}^{\prime}}, \infty\right.$ ). (If $k=1$, there is only a single period $[0, \infty)$.) Exactly one request in $R^{\prime}$, namely $r_{i}^{\prime}$, starts in period $i$, for $1 \leq i \leq k$. We define $R_{i}^{\prime}=\left\{r_{i}^{\prime}\right\}$ for $1 \leq i \leq k$. Let $R_{i}^{*}$ denote the set of requests accepted by $O P T$ that start in period $i$, for $1 \leq i \leq k$.

For $1<i \leq k, r_{i}^{\prime}$ starts at time $t_{r_{i}^{\prime}}$ and the first request of $R_{i}^{*}$ starts during the interval $\left[t_{r_{i}^{\prime}}, t_{r_{i+1}^{\prime}}\right.$ ) (or the interval $\left[t_{r_{k}^{\prime}}, \infty\right.$ ) if $i=k$ ). Furthermore, $r_{1}^{\prime}$ is the first acceptable request in $R$, and so the first request of $R_{1}^{*}$ cannot start before $r_{1}^{\prime}$. Hence, for all $1 \leq i \leq k$, the first request in $R_{i}^{*}$ cannot start before the request $r_{i}^{\prime}$.

We bound the competitive ratio of GA by analyzing each period independently. As $R^{\prime}=\bigcup_{i} R_{i}^{\prime}$ and $R^{*}=\bigcup_{i} R_{i}^{*}$, it is clear that $P_{R^{*}} / P_{R^{\prime}} \leq \alpha$ follows if we can show that $P_{R_{i}^{*}} / P_{R_{i}^{\prime}} \leq \alpha$ for all $i, 1 \leq i \leq k$.

For all $1 \leq i \leq k$, as $R_{i}^{\prime}=\left\{r_{i}\right\}$, we have $P_{R_{i}^{\prime}} \in\{r, r-c\}$. It suffices to show $P_{R_{i}^{*}} / P_{R_{i}^{\prime}} \leq 2 r /(r-c)$ to prove the theorem. We claim that $R_{i}^{*}$ contains at most two requests. Assume that $R_{i}^{*}$ contains at least three requests. Let $r_{j}$ be the third request (in order of start time) in $R_{i}^{*}$. As the first request in $R_{i}^{*}$ does not start before $t_{r_{i}^{\prime}}$, we have $t_{r_{j}} \geq t_{r_{i}^{\prime}}+2 t$. This means that $r_{j}$ would be acceptable to GA after it has accepted $r_{i}^{\prime}$. Therefore, GA accepts either $r_{j}$ or another request starting before $t_{r_{j}}$, and that request becomes $r_{i+1}^{\prime}$. Hence, there cannot be such a request $r_{j}$ that starts in period $i$.

As we have shown that $R_{i}^{*}$ contains at most two requests, we get that $P_{R_{i}^{*}} \leq$ $2 r$. Since $P_{R_{i}^{\prime}} \geq r-c$, we have $P_{R_{i}^{*}} / P_{R_{i}^{\prime}} \leq 2 r /(r-c)$. The theorem follows.

\section{Car Sharing with Variable Booking Times}

In this section, we study the $1 \mathrm{~S} 2 \mathrm{~L}-\mathrm{V}$ problem. Recall that the booking time of a request $r_{i}$ must satisfy $t_{r_{i}}-b_{u} \leq \tilde{t}_{r_{i}} \leq t_{r_{i}}-b_{l}$. First, we present three lower bound results, one for the case $c=r$ and two for the case $c<r$.

Theorem 4. No deterministic algorithm for $1 S 2 L-V$ can have competitive ratio smaller than $1+2\left\lceil\frac{b_{u}-b_{l}}{2 t}\right\rceil$ if $c=r$. In particular, the lower bound is 3 if $0<$ $b_{u}<t$.

Proof. Let $A L G$ be an arbitrary on-line algorithm, and let $O P T$ be an optimal scheduler. We distinguish two cases based on the value of $b_{u}-b_{l}$.

Case 1: $0<b_{u}-b_{l} \leq 2 t$. We need to show that the competitive ratio is at least 3. Define four requests as follows: $r_{1}=\left(\frac{b_{u}+b_{l}}{2}+t, b_{u}+b_{l}+t, 0\right)$, $r_{2}=\left(\frac{2 b_{u}+b_{l}}{3}+t, b_{l}+\frac{2 b_{u}+b_{l}}{3}+t, 0\right), r_{3}=\left(\frac{2 b_{u}+b_{l}}{3}+2 t, b_{l}+\frac{2 b_{u}+b_{l}}{3}+2 t, 1\right), r_{4}=$ $\left(\frac{2 b_{u}+b_{l}}{3}+3 t, b_{l}+\frac{2 b_{u}+b_{l}}{3}+3 t, 0\right)$. Note that a server can accept either $r_{1}$, or all of $r_{2}, r_{3}, r_{4}$. Furthermore, $r_{2}$ is released after $r_{1}$ but starts earlier.

Initially, the adversary releases $r_{1}$. There are two sub-cases. 
Case 1.1: $A L G$ accepts $r_{1}$. The adversary releases $r_{2}, r_{3}$ and $r_{4}$. OPT accepts $r_{2}, r_{3}, r_{4}$ without cost, so we have $P_{R^{*}}=3 r$ and $P_{R^{\prime}}=r$, showing that $P_{R^{*}} / P_{R^{\prime}}=3$.

Case 1.2: $A L G$ does not accept request $r_{1}$. OPT accepts $r_{1}$. We have $P_{R^{*}}=r$ and $P_{R^{\prime}}=0$, and hence $P_{R^{*}} / P_{R^{\prime}}=\infty$.

The lower bound of 3 follows.

Case 2: $2 t<b_{u}-b_{l}$. Let $n=\left\lceil\frac{b_{u}-b_{l}}{2 t}\right\rceil-1$. Choose values $\varepsilon_{i}$ for $1 \leq i \leq n+2$ satisfying $0 \leq \varepsilon_{1}<\varepsilon_{2}<\cdots<\varepsilon_{n+1}<\varepsilon_{n+2}<\min \left\{t, b_{u}-b_{l}-2 t n\right\}$.

Initially, the adversary releases the request sequence $R_{1}$ consisting of the following requests: $r_{1}=\left(\varepsilon_{1}, b_{l}+\varepsilon_{n+2}+t, 1\right), r_{2}=\left(\varepsilon_{2}, b_{l}+\varepsilon_{n+2}+3 t, 1\right), \ldots, r_{i}=$ $\left(\varepsilon_{i}, b_{l}+\varepsilon_{n+2}+(2 i-1) t, 1\right), \ldots, r_{n}=\left(\varepsilon_{n}, b_{l}+\varepsilon_{n+2}+(2 n-1) t, 1\right)$ and $r_{n+1}=$ $\left(\varepsilon_{n+1}, b_{u}+\varepsilon_{n+1}, 0\right)$. Note that $b_{l} \leq b_{l}+\varepsilon_{n+2}+(2 i-1) t \leq b_{u}$ for all $1 \leq i \leq n$. There are three sub-cases.

Case 2.1: $A L G$ rejects all the requests of $R_{1}$. In this case, OPT accepts the request $r_{n+1}$. We have $P_{R^{*}}=r$ and $P_{R^{\prime}}=0$, yielding $P_{R^{*}} / P_{R^{\prime}}=\infty$.

Case 2.2: The first request accepted by $A L G$ is $r_{i}$ for some $i$ with $1 \leq i \leq n$. In this case, the adversary does not release the remaining requests of $R_{1}$. Instead, it releases only one final request $r_{f}=\left(\varepsilon_{i+1}, b_{l}+(2 i-1) t, 0\right)$. ALG cannot accept $r_{f}$ as it is in conflict with $r_{i}$. OPT accepts $r_{f}$. We have $P_{R^{*}}=r$ and $P_{R^{\prime}}=r-c=0$, hence $P_{R^{*}} / P_{R^{\prime}}=\infty$.

Case 2.3: The first request accepted by $A L G$ is $r_{n+1}$. The adversary then releases the request sequence $R_{2}$ consisting of the following requests: $r_{n+1+1}=$ $\left(\varepsilon_{n+2}, b_{l}+\varepsilon_{n+2}, 0\right), r_{n+1+2}=\left(\varepsilon_{n+2}, b_{l}+\varepsilon_{n+2}+2 t, 0\right), \ldots, r_{n+1+i}=\left(\varepsilon_{n+2}, b_{l}+\right.$ $\left.\varepsilon_{n+2}+2(i-1) t, 0\right), \ldots, r_{n+1+n}=\left(\varepsilon_{n+2}, b_{l}+\varepsilon_{n+2}+2(n-1) t, 0\right)$. After this, the adversary releases the request sequence $R_{3}$ consisting of three more requests: $r_{2 n+1+1}=\left(\varepsilon_{n+2}, b_{u}, 0\right), r_{2 n+1+2}=\left(\varepsilon_{n+2}+t, b_{u}+t, 1\right), r_{2 n+1+3}=\left(\varepsilon_{n+2}+\right.$ $\left.2 t, b_{u}+2 t, 0\right)$. $A L G$ cannot accept any requests of $R_{3}$ as they all conflict with $r_{n+1}$. $A L G$ can accept any number of requests of $R_{2}$, but since they all have pick-up location 0 (as does $r_{n+1}$ ), its total profit will be $P_{R^{\prime}}=r$. OPT accepts all requests of $R_{1}$ except $r_{n+1}$, and all requests of $R_{2}$ and $R_{3}$. We have $P_{R^{*}}=$ $(2 n+3) r=\left(2\left\lceil\frac{b_{u}-b_{l}}{2 t}\right\rceil+1\right) r$. Hence, $P_{R^{*}} / P_{R^{\prime}}=1+2\left\lceil\frac{b_{u}-b_{l}}{2 t}\right\rceil$.

The claimed lower bound of $1+2\left\lceil\frac{b_{u}-b_{l}}{2 t}\right\rceil$ follows.

Theorem 5. No deterministic algorithm for $1 S 2 L-V$ can have competitive ratio smaller than $\frac{3 r-c}{r-c}$ if $0 \leq c<r$ and $b_{u}>t$.

Proof. Initially, the adversary releases the request $r_{1}=\left(0, b_{u}, 1\right)$. We distinguish two cases.

Case 1: $A L G$ accepts $r_{1}$ (with cost). The adversary releases requests $r_{2}=$ $\left(\varepsilon, t_{r_{1}}-\varepsilon, 1\right), r_{3}=\left(\varepsilon+t, t_{r_{2}}+t, 0\right)$ and $r_{4}=\left(\varepsilon+2 t, t_{r_{3}}+t, 1\right)$, where $0<$ $\varepsilon<\min \left\{t, \frac{b_{u}-b_{l}}{2}\right\}$. OPT accepts $r_{2}, r_{3}$ and $r_{4}$. As they are in conflict with $r_{1}$, $A L G$ cannot accept any of them, see also Fig. 1. We have $P_{R^{*}}=3 r-c$ and $P_{R^{\prime}}=r-c$. Hence, $P_{R^{*}} / P_{R^{\prime}}=\frac{3 r-c}{r-c}$.

Case 2: $A L G$ does not accept request $r_{1}$. In this case, OPT accepts $r_{1}$. We have $P_{R^{*}}=r-c, P_{R^{\prime}}=0$, and hence $P_{R^{*}} / P_{R^{\prime}}=\infty$. 


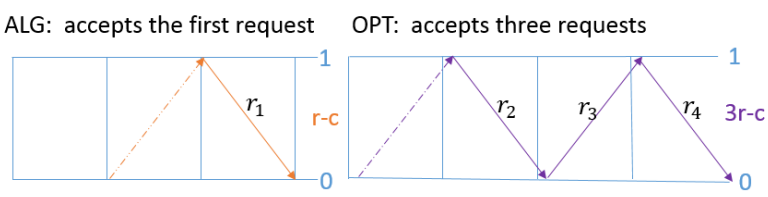

Fig. 1. Case 1: $\frac{P_{R^{*}}}{P_{R^{\prime}}}=\frac{3 r-c}{r-c}$

Theorem 6. No deterministic algorithm for $1 S 2 L-V$ can have competitive ratio smaller than 3 if $0 \leq c<r$ and $b_{u} \leq t$.

Proof. Initially, the adversary releases the request $r_{1}=\left(0, b_{u}, 0\right)$. We distinguish two cases.

Case 1: $A L G$ accepts $r_{1}$ (without cost). The adversary releases requests $r_{2}=\left(\varepsilon, t_{r_{1}}-\varepsilon, 0\right), r_{3}=\left(\varepsilon+t, t_{r_{2}}+t, 1\right)$ and $r_{4}=\left(\varepsilon+2 t, t_{r_{3}}+t, 0\right)$, where $0<\varepsilon<\min \left\{t, \frac{b_{u}-b_{l}}{2}\right\}$. OPT accepts $r_{2}, r_{3}$ and $r_{4}$. As they are in conflict with $r_{1}, A L G$ cannot accept any of them. We have $P_{R^{*}}=3 r$ and $P_{R^{\prime}}=r$. Hence, $P_{R^{*}} / P_{R^{\prime}}=3$.

Case 2: $A L G$ does not accept request $r_{1}$. In this case, $O P T$ accepts $r_{1}$. We have $P_{R^{*}}=r, P_{R^{\prime}}=0$, and hence $P_{R^{*}} / P_{R^{\prime}}=\infty$.

From Theorems 1 and 6 we can conclude that no deterministic algorithm for $1 \mathrm{~S} 2 \mathrm{~L}-\mathrm{V}$ can have competitive ratio smaller than $\max \left\{\frac{2 r}{r-c}, 3\right\}$ if $0 \leq c<r$ and $b_{u}=t$.

We now turn to upper bounds and analyze Algorithm GA (which was presented as Algorithm 1 in Section 2) for the $1 \mathrm{~S} 2 \mathrm{~L}-\mathrm{V}$ problem. Denote the set of requests accepted by $O P T$ by $R^{*}$ and the set of requests accepted by GA as $R^{\prime}$. The server of $O P T$ is referred to as $s^{*}$, and the server of GA as $s^{\prime}$. Let $R^{\prime}=\left\{r_{1}^{\prime}, \ldots, r_{k}^{\prime}\right\}$, with the requests indexed in order of increasing start time. For $1 \leq i \leq k$, let $R_{i}^{\prime}=\left\{r_{i}^{\prime}\right\}$. We partition the time horizon $[0, \infty)$ into intervals (periods) that can be analyzed independently. The partition differs for GA and $O P T$, so we refer to GA periods and OPT periods. GA period $i$ is the interval $\left[0, t_{r_{2}^{\prime}}\right)$ if $i=1$, the interval $\left[t_{r_{k}^{\prime}}, \infty\right)$ if $i=k$, and the interval $\left[t_{r_{i}^{\prime}}, t_{r_{i+1}^{\prime}}\right)$ if $1<i<k$. Note that $R_{i}^{\prime}$ consists of the only request in $R^{\prime}$ that starts in GA period $i$.

For $1 \leq i \leq k$, define $\hat{t}_{r_{i}^{\prime}}$ to be the first time when the optimal server $s^{*}$ is at location $\dot{p}_{r_{i}^{\prime}}$ at or after time $\dot{t}_{r_{i}^{\prime}}$, or $\infty$ if $s^{*}$ never reaches $\dot{p}_{r_{i}^{\prime}}$ from time $\dot{t}_{r_{i}^{\prime}}$ onward. Now, define $O P T$ period $i$ to be the interval $\left[0, \hat{t}_{r_{1}^{\prime}}\right)$ if $i=1$, the interval $\left[\hat{t}_{k-1}, \infty\right)$ if $i=k$, and the interval $\left[\hat{t}_{r_{i-1}^{\prime}}, \hat{t}_{r_{i}^{\prime}}\right)$ if $1<i<k$. For $1 \leq i \leq k$, let $R_{i}^{*}$ be the set of requests accepted by $O P T$ that start during $O P T$ period $i$. If $\hat{t}_{r_{i}^{\prime}} \leq \hat{t}_{r_{i-1}^{\prime}}, O P T$ period $i$ is empty and $R_{i}^{*}=\emptyset$. The $j^{\text {th }}$ (in order of start times) request of $R_{i}^{*}$ is denoted by $R_{i}^{*}(j)$.

We will compare the profit $P_{R_{i}^{\prime}}$ that GA accrues in GA period $i$ with the profit $P_{R_{i}^{*}}$ that $O P T$ accrues in $O P T$ period $i$. We can again analyze each period independently: If we can show that $P_{R_{i}^{*}} / P_{R_{i}^{\prime}} \leq \alpha$ for all $i$, this implies that $P_{R^{*}} / P_{R^{\prime}} \leq \alpha$. We first state two observations. 
Observation 1 For all $i, s^{\prime}$ is at $\dot{p}_{r_{i}^{\prime}}$ at time $\dot{t}_{r_{i}^{\prime}}$, and $s^{*}$ is at $\dot{p}_{r_{i}^{\prime}}$ at time $\hat{t}_{r_{i}^{\prime}}$, and $\hat{t}_{r_{i}^{\prime}} \geq \dot{t}_{r_{i}^{\prime}}$

Observation 2 If $c=r$, by definition of Algorithm GA (Algorithm 1), for $1<i \leq k$ we have that $\tilde{t}_{r_{i-1}^{\prime}} \leq \tilde{t}_{r_{i}^{\prime}}$ (otherwise, $P_{R_{i}^{G A} \cup\left\{r_{i}^{\prime}\right\}}-P_{R_{i}^{G A}}=0$ and $r_{i}^{\prime}$ would be rejected).

Theorem 7. Algorithm GA is 3-competitive for $1 S 2 L-V$ if $0<b_{u}<t$ and $0 \leq c<r$.

Proof. Due to $0<b_{u}<t$, all requests of $R_{i}^{*}$ and $R_{i}^{\prime}$ must be accepted without cost because the request arrival is too late to serve a request with cost (recall that we forbid unprompted moves by $O P T$ ).

First, consider period $i=1$. OPT cannot accept any request that is released during the time interval $\left[0, \tilde{t}_{r_{1}^{\prime}}\right)$, because otherwise such a request accepted by OPT could be accepted by GA instead of $r_{1}^{\prime}$. Thus $\tilde{t}_{R_{1}^{*}(1)} \geq \tilde{t}_{r_{1}^{\prime}}$, and hence $t_{R_{1}^{*}(1)} \geq \tilde{t}_{r_{1}^{\prime}} \geq t_{r_{1}^{\prime}}-b_{u}$. By Observation 1 and because OPT does not accept any request with cost, $\dot{p}_{R_{i}^{*}(1)}=\dot{p}_{r_{1}^{\prime}}, \dot{p}_{R_{1}^{*}(2)}=p_{r_{1}^{\prime}}$, and $\dot{p}_{R_{1}^{*}(3)}=\dot{p}_{r_{1}^{\prime}}$. Hence, $s^{*}$ is at $\dot{p}_{r_{1}^{\prime}}$ at time $\dot{t}_{R_{i}^{*}(3)}$, which is not before $\dot{t}_{r_{1}^{\prime}}$ (because $t_{R_{1}^{*}(3)} \geq t_{R_{1}^{*}(1)}+2 t \geq$ $\left.t_{r_{1}^{\prime}}-b_{u}+2 t>t_{r_{1}^{\prime}}+t\right)$. Therefore, $\left|R_{1}^{*}\right| \leq 3$. Thus, $P_{R_{1}^{*}} / P_{R_{1}^{\prime}} \leq 3$.

For $1<i \leq k$, we distinguish the following cases in order to bound $P_{R_{i}^{*}} / P_{R_{i}^{\prime}}$. As $R_{i}^{\prime}=\left\{r_{i}^{\prime}\right\}, P_{R_{i}^{\prime}}=r$. We need to show that $P_{R_{i}^{*}} \leq 3 r$.

Case 1: $\hat{t}_{r_{i-1}^{\prime}}<\hat{t}_{r_{i}^{\prime}}$ and $\hat{t}_{r_{i-1}^{\prime}}<t_{r_{i}^{\prime}}$. Assume that $\left|R_{i}^{*}\right| \geq 3$. (Otherwise, there is nothing to show.) By Observation 1 and because $O P T$ does not accept any request with cost, $\dot{p}_{R_{i}^{*}(1)}=\dot{p}_{r_{i}^{\prime}}, \dot{p}_{R_{i}^{*}(2)}=p_{r_{i}^{\prime}}$, and $\dot{p}_{R_{i}^{*}(3)}=\dot{p}_{r_{i}^{\prime}}$. If $t_{R_{i}^{*}(1)} \geq t_{r_{i}^{\prime}}$, we have $\hat{t}_{r_{i}^{\prime}}=\dot{t}_{R_{i}^{*}(1)}$ and thus $\left|R_{i}^{*}\right|=1$. Therefore, we must have $t_{R_{i}^{*}(1)}<t_{r_{i}^{\prime}}$. Observe that $O P T$ can only accept requests with pick-up location $p_{r_{i}^{\prime}}$ that start before $t_{r_{i}^{\prime}}$ if they start after $t_{r_{i}^{\prime}}-b_{u}>t_{r_{i}^{\prime}}-t$. Otherwise, such a request accepted by $O P T^{i}$ would arrive before $r_{i}^{\prime}$ and so it would be accepted by GA instead of $r_{i}^{\prime}$. So we have $\dot{t}_{R_{i}^{*}(3)} \geq t_{R_{i}^{*}(1)}+3 t \geq t_{r_{i}^{\prime}}-b_{u}+3 t>\dot{t}_{r_{i}^{\prime}}$. When OPT finishes serving the third request of $R_{i}^{*}, s^{*}$ is at $\dot{p}_{r_{i}^{\prime}}$, and this happens at a time after $\dot{t}_{r_{i}^{\prime}}$ (see Fig. 2 for an illustration). Therefore, this time is $\hat{t}_{r_{i}^{\prime}}$ and thus the end of $O P T$ period $i$, and $R_{i}^{*}$ cannot contain any further requests. (If $i=k$, the end of $O P T$ period $i$ is $\infty$, but any further request accepted by $O P T$ could also be accepted by GA, a contradiction.) We have shown $\left|R_{i}^{*}\right| \leq 3$, as required. Hence $P_{R_{i}^{*}} / P_{R_{i}^{\prime}} \leq 3$

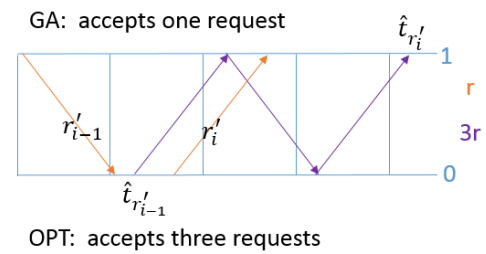

Fig. 2. Example configuration for $R_{i}^{*}$ and $R_{i}^{\prime}$ in Case 1 
Case 2: $\hat{t}_{r_{i-1}^{\prime}}<\hat{t}_{r_{i}^{\prime}}$ and $\hat{t}_{r_{i-1}^{\prime}} \geq t_{r_{i}^{\prime}}$. We claim that $\left|R_{i}^{*}\right| \leq 1$ and argue as follows. Because $\hat{t}_{r_{i-1}^{\prime}} \geq t_{r_{i}^{\prime}}, O P T$ can accept at most one request in $O P T$ period $i$ : When $s^{*}$ finishes serving the first request in $R_{i}^{*}, s^{*}$ is located at $\dot{p}_{r_{i}^{\prime}}$, and the time when this happens becomes $\hat{t}_{r_{i}^{\prime}}$ and thus the end of $O P T$ period $i$. (If $i=k$, we can argue as in Case 1 that $O P T$ cannot accept any further requests.) Hence, $P_{R_{i}^{*}} / P_{R_{i}^{\prime}} \leq 1<3$.

Case 3: $\hat{t}_{r_{i-1}^{\prime}} \geq \hat{t}_{r_{i}^{\prime}}$. As $O P T$ period $i$ is empty by definition, we have $R_{i}^{*}=\emptyset$ and hence $P_{R_{i}^{*}}=0$. Thus, $P_{R_{i}^{*}} / P_{R_{i}^{\prime}}<3$.

Because $P_{R_{i}^{*}} / P_{R_{i}^{\prime}} \leq 3$ holds for all $1 \leq i \leq k$, we have $P_{R^{*}} / P_{R^{\prime}} \leq 3$. This proves the theorem.

Theorem 8. Algorithm GA is $\max \left\{\frac{2 r}{r-c}, 3\right\}$-competitive for $1 S 2 L-V$ if $0 \leq c<r$ and $b_{u}=t$.

Theorem 9. Algorithm GA is $\frac{3 r-c}{r-c}$-competitive for 1S2L-V if $0 \leq c<r$ and $b_{u}>t$.

The proofs of Theorems 8 and 9 are omitted due to space restrictions.

Theorem 10. Algorithm GA has competitive ratio at most $1+2\left\lceil\frac{b_{u}-b_{l}}{2 t}\right\rceil$ for $1 S 2 L-V$ if $c=r$. In particular, it is 3-competitive if $0<b_{u}<t$.

Proof. We prove the theorem by induction over the size of $R^{\prime}$.

Base Case: $\left|R^{\prime}\right|=1$. We can show that $\left|R^{*}\right| \leq 1+2 \cdot\left\lceil\frac{b_{u}-b_{l}}{2 t}\right\rceil$. As $P_{R^{\prime}}=r$ and $P_{R^{*}}=\left|R^{*}\right| \cdot r$, we get $P_{R^{*}} / P_{R^{\prime}} \leq 1+2\left\lceil\frac{b_{u}-b_{l}}{2 t}\right\rceil$. The arguments are similar to those used in the Induction Step below and are omitted here.

Induction Step: We assume that $\frac{P_{R^{*}}}{P_{R^{\prime}}} \leq 1+2\left\lceil\frac{b_{u}-b_{l}}{2 t}\right\rceil$ holds for all instances with $\left|R^{\prime}\right| \leq i$ and show that then $\frac{P_{R^{*}}}{P_{R^{\prime}}} \leq 1+2\left\lceil\frac{b_{u}-b_{l}}{2 t}\right\rceil$ also holds for all instances with $\left|R^{\prime}\right|=i+1$.

Consider an instance of $1 \mathrm{~S} 2 \mathrm{~L}-\mathrm{V}$ given by a request sequence $R$ where GA accepts $i+1$ requests. As GA accepts requests in order of increasing arrival time by Observation 2, GA accepts $i$ requests before time $\tilde{t}_{r_{i+1}^{\prime}}$. Let $\bar{R}$ be the sub-instance of $R$ that contains all requests in $R$ except $r_{i+1}^{\prime}$ and all requests that are released after $r_{i+1}^{\prime}$ (i.e., released at time $\tilde{t}_{r_{i+1}^{\prime}}$ and processed after $r_{i+1}^{\prime}$, or released after time $\tilde{t}_{r_{i+1}^{\prime}}$ ) and that GA could accept instead of $r_{i+1}^{\prime}$. By the inductive hypothesis, $O P T$ can achieve profit at most $i \cdot\left(1+2\left\lceil\frac{b_{u}-b_{l}}{2 t}\right\rceil\right) \cdot r$ on the request sequence $\bar{R}$. The increase in profit that $O P T$ can achieve on request sequence $R$ compared to $\bar{R}$ must be due to requests accepted without cost that start in the interval $\left[\tilde{t}_{r_{i+1}^{\prime}}+b_{l}, \infty\right)$ as all requests that start earlier were presented before time $\tilde{t}_{r_{i+1}^{\prime}}$ and are thus contained in $\bar{R}$. Let $Q$ be a largest set of requests in $R \backslash \bar{R}$ with start times in $\left[\tilde{t}_{r_{i+1}^{\prime}}+b_{l}, \infty\right)$ that can be accepted without cost by $O P T$. Clearly, $P_{R^{*}} \leq P_{\bar{R}^{*}}+r \cdot|Q|$, where $\bar{R}^{*}$ denotes the requests accepted by an optimal solution for the instance $\bar{R}$.

We claim that the first request in $Q$ must have pick-up location $p_{r_{i+1}^{\prime}}$ as otherwise that request would have to be contained in $\bar{R}$, a contradiction to $Q$ 
being a subset of $R \backslash \bar{R}$. To see this, assume that the request with earliest start time after $\tilde{t}_{r_{i+1}^{\prime}}+b_{l}$ in $Q$ has pick-up location $\dot{p}_{r_{i+1}^{\prime}}$. Denote that request by $r_{j}$. If $r_{j}$ was presented before $r_{i+1}^{\prime}$, it is clearly contained in $\bar{R}$. If $r_{j}$ was presented after $r_{i+1}^{\prime}$, it is also contained in $\bar{R}$ because GA cannot accept it instead of $r_{i+1}^{\prime}$ (as $s^{\prime}$ is at location $p_{r_{i+1}^{\prime}}$ after serving $r_{i}^{\prime}$ and GA accepts requests in order of increasing start times by Observation 2).

Before time $\tilde{t}_{r_{i+1}^{\prime}}, O P T$ can accept requests with pick-up location $\dot{p}_{r_{i+1}^{\prime}}$ that start no later than $\tilde{t}_{r_{i+1}^{\prime}}+b_{u}$. After time $\tilde{t}_{r_{i+1}^{\prime}}$, OPT can only accept requests with pick-up location $\dot{p}_{r_{i+1}^{\prime}}$ that start strictly before $t_{r_{i+1}^{\prime}}+t$, because $s^{\prime}$ arrives at $\dot{p}_{r_{i+1}^{\prime}}$ at time $t_{r_{i+1}^{\prime}}+t$ and could serve any request with pick-up location $\dot{p}_{r_{i+1}^{\prime}}$ from that time onward.

First, consider the case that $\tilde{t}_{r_{i+1}^{\prime}}+b_{u} \geq t_{r_{i+1}^{\prime}}+t$. The last request that OPT can accept with pick-up location $\dot{p}_{r_{i+1}^{\prime}}$ starts no later than $\tilde{t}_{r_{i+1}^{\prime}}+b_{u}$. After that request, OPT can accept at most one more request with pick-up location $p_{r_{i+1}^{\prime}}$. To bound the size of $Q$, we bound the maximum number of pairs of requests (one with pick-up location 0 and the next with pick-up location 1 ) that $O P T$ can accept. As the last request with pick-up location $\dot{p}_{r_{i+1}^{\prime}}$ that $O P T$ can accept has start time at most $\tilde{t}_{r_{i+1}^{\prime}}+b_{u}$, the start time of the first request of the last pair that $O P T$ accepts is at most $\tilde{t}_{r_{i+1}^{\prime}}+b_{u}-t$. As the start times of consecutive pairs differ by at least $2 t$, the number of pairs is bounded by $1+\left\lfloor\left(\left(\tilde{t}_{r_{i+1}^{\prime}}+b_{u}-t\right)-\left(\tilde{t}_{r_{i+1}^{\prime}}+b_{l}\right)\right) / 2 t\right\rfloor=1+\left\lfloor\left(b_{u}-b_{l}-t\right) / 2 t\right\rfloor$. If $b_{u}-b_{l}-t$ is a multiple of $2 t$, this bound is equal to $1+\left(b_{u}-b_{l}-t\right) / 2 t=\left\lceil\left(b_{u}-b_{l}\right) / 2 t\right\rceil$. Otherwise, the bound is equal to $\left\lceil\left(b_{u}-b_{l}-t\right) / 2 t\right\rceil \leq\left\lceil\left(b_{u}-b_{l}\right) / 2 t\right\rceil$. After the last pair, OPT can accept at most one more request with pick-up location $p_{r_{i+1}^{\prime}}$. Therefore, $|Q| \leq 1+2 \cdot\left\lceil\frac{b_{u}-b_{l}}{2 t}\right\rceil$.

Now, consider the case that $\tilde{t}_{r_{i+1}^{\prime}}+b_{u}<t_{r_{i+1}^{\prime}}+t$. Again, we consider the maximum number of pairs of requests (one with pick-up location $p_{r_{i+1}^{\prime}}$ and the next with pick-up location $\left.\dot{p}_{r_{i+1}^{\prime}}\right)$ that $O P T$ can accept. As the last request with pick-up location $\dot{p}_{r_{i+1}^{\prime}}$ that $O P T$ can accept must have start time strictly smaller than $t_{r_{i+1}^{\prime}}+t \leq \tilde{t}_{r_{i+1}^{\prime}}+b_{u}+t$, the start time of the first request of the last pair that $O P T$ accepts is strictly smaller than $\tilde{t}_{r_{i+1}^{\prime}}+b_{u}$. The start times of consecutive pairs differ by at least $2 t$. If $b_{u}-b_{l}$ is a multiple of $2 t$, the number of pairs is bounded by $\left(\left(\tilde{t}_{r_{i+1}^{\prime}}+b_{u}\right)-\left(\tilde{t}_{r_{i+1}^{\prime}}+b_{l}\right)\right) / 2 t=\left(b_{u}-b_{l}\right) / 2 t=\left\lceil\left(b_{u}-b_{l}\right) / 2 t\right\rceil$. If $b_{u}-b_{l}$ is not a multiple of $2 t$, the number of pairs is bounded by $1+\left\lfloor\left(b_{u}-b_{l}\right) / 2 t\right\rfloor=$ $\left\lceil\left(b_{u}-b_{l}\right) / 2 t\right\rceil$. In any case, the number of pairs is at most $\left\lceil\left(b_{u}-b_{l}\right) / 2 t\right\rceil$. After the last pair, OPT can accept at most one more request with pick-up location $p_{r_{i+1}^{\prime}}$. Therefore, $|Q| \leq 1+2 \cdot\left\lceil\frac{b_{u}-b_{l}}{2 t}\right\rceil$.

In either case, $|Q| \leq 1+2\left\lceil\frac{b_{u}-b_{l}}{2 t}\right\rceil$. Thus, $P_{R^{*}} \leq P_{\bar{R}^{*}}+r \cdot|Q| \leq i(1+$ $\left.2\left\lceil\frac{b_{u}-b_{l}}{2 t}\right\rceil\right) r+\left(1+2\left\lceil\frac{b_{u}-b_{l}}{2 t}\right\rceil\right) r=(i+1)\left(1+2\left\lceil\frac{b_{u}-b_{l}}{2 t}\right\rceil\right) r$. As $P_{R^{\prime}}=(i+1) r$, we get $P_{R^{*}} / P_{R^{\prime}} \leq 1+2\left\lceil\frac{b_{u}-b_{l}}{2 t}\right\rceil$. 


\section{Conclusion}

We have studied an on-line problem with one server and two locations that is motivated by applications such as car sharing and taxi dispatching. In particular, we have analyzed the effects of different constraints on the booking time of requests on the competitive ratio that can be achieved. For all variants of booking time constraints and costs for empty server movements we have given matching lower and upper bounds on the competitive ratio. The upper bounds are all achieved by the same greedy algorithm (GA). Interestingly, the size of the booking horizon does not affect the competitive ratio if $0 \leq c<r$, but the competitive ratio increases as $b_{u}-b_{l}$ increases if $c=r$.

A number of directions for future work arise from this work. In particular, it would be interesting to extend our results to the case of more than one server and more than two locations. It would be interesting to determine how the constraints on the booking time affect the competitive ratio for the general carsharing problem with $k$ servers and $m$ locations.

Acknowledgments. This work was partially supported by the China Postdoctoral Science Foundation (Grant No. 2016M592811), and the China Scholarship Council (Grant No. 201706280058).

\section{References}

1. Ascheuer, N., Krumke, S.O., Rambau, J.: Online dial-a-ride problems: Minimizing the completion time. In: STACS 2000, LNCS, vol. 1770, pp. 639-650. Springer (2000)

2. Berbeglia, G., Cordeau, J., Laporte, G.: Dynamic pickup and delivery problems. European Journal of Operational Research 202(1), 8-15 (2010)

3. Bjelde, A., Disser, Y., Hackfeld, J., Hansknecht, C., Lipmann, M., Meißner, J., Schewior, K., Schlöter, M., Stougie, L.: Tight bounds for online TSP on the line. In: SODA 2017, pp. 994-1005. SIAM (2017)

4. Böhmová, K., Disser, Y., Mihalák, M., Srámek, R.: Scheduling transfers of resources over time: Towards car-sharing with flexible drop-offs. In: LATIN 2016, LNCS, vol. 9644, pp. 220-234. Springer (2016)

5. Borodin, A., El-Yaniv, R.: Online computation and competitive analysis. Cambridge University Press (1998)

6. Christman, A., Forcier, W., Poudel, A.: From theory to practice: maximizing revenues for on-line dial-a-ride. J. Comb. Optim. 35(2), 512-529 (2018)

7. Kaushik, N.R., Figueira, S.M., Chiappari, S.A.: Flexible time-windows for advance reservation scheduling. In: MASCOTS 2006, pp. 218-225. IEEE Computer Society (2006)

8. Krumke, S.O., de Paepe, W., Poensgen, D., Lipmann, M., Marchetti-Spaccamela, A., Stougie, L.: On minimizing the maximum flow time in the online dial-a-ride problem. In: WAOA 2006, LNCS, vol. 3879, pp. 258-269. Springer (2006)

9. Yi, F., Tian, L.: On the online dial-a-ride problem with time-windows. In: AAIM 2005, LNCS, vol. 3521, pp. 85-94. Springer (2005) 